\title{
Proteomics-based identification of a tumor-associated antigen and its corresponding autoantibody in gastric cancer
}

\author{
SOICHIRO TSUNEMI ${ }^{1}$, TOYOFUMI NAKANISHI ${ }^{2}$, YOSHIHISA FUJITA ${ }^{1}$, \\ GEORGE BOURAS $^{1}$, YOSHIHARU MIYAMOTO ${ }^{1}$, AKIKO MIYAMOTO ${ }^{1}$, \\ EIJI NOMURA $^{1}$, TAKAYUKI TAKUBO ${ }^{2}$ and NOBUHIKO TANIGAWA ${ }^{1}$
}

\begin{abstract}
Departments of ${ }^{1}$ General and Gastroenterological Surgery and ${ }^{2}$ Clinical Pathology, Osaka Medical College, 2-7 Daigaku-machi, Takatsuki-city, Osaka 569-8686, Japan
\end{abstract}

Received November 26, 2009; Accepted December 29, 2009

DOI: $10.3892 /$ or_00000719

\begin{abstract}
Identification of novel tumor-related antigens and autoantibodies will lead to early diagnosis of cancer and the development of more effective immunotherapies. The purpose of this study was to identify novel tumor antigens from the gastric cancer cell lines MkN-1, MkN-45 and KATOIII, and their related autoantibodies in sera of patients with gastric cancer using a proteomics-based approach. Proteins from the gastric cancer cell lines $(\mathrm{MkN}-1, \mathrm{MkN}-45$ and KATOIII) were separated by two-dimensional polyacrylamide gel electrophoresis, followed by Western blotting and antibody reaction with sera from patients with gastric cancer, healthy individuals and patients with other cancers. Positive spots were excised from Coomassie blue stained gels and analyzed by matrix-assisted laser desorption/ionization time-of-flight mass sprctrometry (MALDI-TOF/TOF MS). Sera from patients with gastric cancer yielded multiple spots, one of which was identified as the $78 \mathrm{kDa}$ glucoseregulated protein (GRP78) by MALDI-TOF/TOF MS. Western blots against recombinant GRP78 showed reactivity in sera from $17 / 60(28.3 \%)$ patients with gastric cancer and $0 / 20(0.0 \%)$ of healthy individuals. Autoantibodies against GRP78 were found in 4/15 (26.7\%) and 3/15 (20.0\%) patients with esophageal and colon cancer, respectively. We identified for the first time an autoantibody against GRP78 in gastric cancer patients. The proteomic approach implemented in this study offers a powerful tool for identifying novel serum markers that may display clinical usefulness in cancer.
\end{abstract}

Correspondence to: Dr Nobuhiko Tanigawa, Department of General and Gastroenterological Surgery, Osaka Medical College, 2-7 Daigaku-machi, Takatsuki-city, Osaka 569-8686, Japan

E-mail: sur001@poh.osaka-med.ac.jp

Key words: 78 kDa glucose-regulated protein, autoantibody, proteomics, gastric cancer, matrix-assisted laser desorption/ ionization-time-of-flight mass spectrometry

\section{Introduction}

Gastric cancer is one of the most frequent malignancies of the gastrointestinal tract in South East Asia and South America. In Japan, gastric cancer represents the second commonest malignancy in both men and women. Although the overall prognosis has improved in recent years because of improvements in diagnosis and treatment, outcomes from advanced disease is still poor with a 5 -year survival rate of $<20 \%$ in stage IV disease. Early detection can therefore lead to improved survival. Currently, the most reliable method of diagnosing gastric cancer is gastroscopic biopsy although this is an uncomfortable and invasive procedure for screening purposes. Tumor markers with high specificity and sensitivity are desirable for screening and early diagnosis of gastric cancer.

In the post-genomic era, the rapid evolution of proteomics research has opened new horizons because it promises to accelerate the discovery of new disease markers. In 2001, Brichory et al (1) introduced a proteomics-based approach to identify tumor antigens and related autoantibodies in cancer. So far, this method has revealed many novel autoantibodies in several malignancies. Autoantibodies against triosephosphate isomerase, superoxide dismutase, Annexin I and II and PGP 9.5 have been reported as useful markers in lung cancer $(1,2)$. Autoantibodies against heat-shock protein 70 (HSP70), peroxiredoxin, Mn-SOD, DEAD (Asp-Glu-Ala-Asp) box polypeptide 3 , eukaryotic translation elongation factor 2 (eEF2), apoptosis-inducing factor (AIE), heterogeneous nuclear ribonucleoprotein A2 (hnRNP A2), prostatic binding protein and triosephosphate isomerase (TIM) have been more frequent in patients with hepatocellular carcinoma $(3,4)$. Similarly, autoantibodies against peroxiredoxin VI and HSP70 have been reported as potential diagnostic markers in esophageal squamous cell carcinoma $(5,6)$. A number of autoantigens have also been reported in breast cancer using serological screening of tumor-derived cDNA expression libraries and phage display libraries (7), although a proteomic approach was used to identify autoantibodies against an oncogenic protein RS/DJ-1 which has been reported as a potential diagnostic marker (8). CEA (cercinoembryonic antigen) and CA19-9 are useful in gastric cancer, however, the lack of specificity and sensitivity of both these markers preclude their general clinical 
use for early disease detection. In contrast, immune responses to tumor proteins may be present early during tumor progression. Analysis of serum autoantibodies may therefore lead to the detection of clinically useful tumor markers.

In the present study, we have applied a proteomics-based approach to identify tumor antigens that induce a humoral immune response in patients with gastric cancer. Cellular proteins from gastric cancer cell lines were separated by 2-D electrophoresis, transferred onto polyvinylidene defluoride (PVDF) membranes and then sera from gastric cancer patients and controls were screened by Western blotting for antibodies that react against separated proteins. Proteins eliciting an immune reaction were isolated and subsequently identified by mass spectrometry. Using this methodology a number of tumor antigens were found to react more frequently with gastric cancer patient sera compared to controls. These identified biomarkers are interesting candidates for screening and early diagnosis of gastric cancer.

\section{Materials and methods}

Sera and cell lines. Sera and tumor tissues were obtained at the time of diagnosis after obtaining written informed consent. The experimental protocol was approved by the Institutional Review Board of Osaka Medical College. Sera were obtained from 60 patients with gastric cancer. Sera from 20 healthy individuals and from 30 patients with other cancers (esophageal cancer, $\mathrm{n}=15$; colon cancer, $\mathrm{n}=15$ ) were used as controls. Mean age of subjects who donated sera for this study was 69.1 years (range, 46-89 years). Gastric cancer was stage I in 27 patients, stage II in 3 patients, stage III in 21 patients, stage IV in 9 patients. The staging for each gastric cancer was evaluated according to the Japanese Classification of Gastric Carcinoma (13th Edition, by Japanese Gastric Cancer Association). Gastric cancer cell lines (MkN-1, MkN-45 and KATOIII) were obtained from Riken BioResource Center (Tsukuba, Japan) and characterized by the Short Tandem RepeatPolymerase Chain Reaction method at the institute. The cell lines (MkN-1, MkN-45, KATOIII) were cultured in RPMI1640 supplemented with $10 \%$ fetal bovine serum (Nikken Cell Media, Kyoto, Japan). Cells were harvested in $300 \mu \mathrm{l}$ of solubilization buffer ( $9 \mathrm{~mol} / 1$ urea, $2 \%$ NP40, 2\% Bmercaptoethanol, $10 \mathrm{mmol} / 1$ phenylmethylsulfonyl fluoride) using a cell scraper and stored at $-80^{\circ} \mathrm{C}$ until use.

Tumor tissue samples. Gastric cancer tissue and paired adjacent normal gastric tissue were obtained during surgical resection from Osaka Medical College Hospital. After excision, sample pieces of tissue were frozen immediately at $-80^{\circ} \mathrm{C}$ and stored until use.

Two-dimensional polyacrylamide gel electrophoresis and Western blotting. To detect autoantibodies against tumor antigens in sera from cancer patients, we followed the approach established by Brichory et al (1). Proteins (30 $\mu \mathrm{g})$ from a mixture of the cell lines (MkN-1, MkN-45 and KATOIII) solubilized from cultured cells underwent isoelectric focusing (IEF) gel electrophoresis (one-dimension electrophoresis) and were then subjected to SDS-PAGE (two-dimension electrophoresis). IEF was conducted with immobilized pH 3.0 to 10.0 non-linear gradient strips (GE Healthcare UK) at 500 $\mathrm{V}$ for $45 \mathrm{~min}, 1,000 \mathrm{~V}$ for $45 \mathrm{~min}$ followed by $5,000 \mathrm{~V}$ for an additional 90 min using Ettan IPGphor II (GE Healthcare UK). After this, the gel strips were equilibrated with $50 \mathrm{mmol} / \mathrm{l}$ Tris (pH 6.8) containing 10\% glycerol, $2 \%$ SDS, $1 \%$ dithiothreitol (DTT) and bromophenol blue and then loaded onto two-dimensional gels. Separated proteins were transferred onto a Hybond P PVDF membrane (GE Healthcare UK) for $1 \mathrm{~h}$ at a constant voltage of $20 \mathrm{~V}$ using a Mini Trans-Blot system (Bio-Rad, Hercules, CA) or visualized by Coomassie Brilliant Blue staining. After transfer, PVDF membranes were incubated with blocking buffer consisting of PBS and 3\% skimmed milk overnight at $4^{\circ} \mathrm{C}$, then washed with washing buffer (PBS/0.05\% Tween-20) and incubated with diluted sera from patients with cancer and healthy subjects at 1:200 for $2 \mathrm{~h}$ at room temperature. After four washings, membranes were reacted with horseradish peroxidase-conjugated mouse antihuman immunoglobulin IgG (GE Healthcare UK) at 1:2000 dilution for $1 \mathrm{~h}$ at room temperature and then washed again. Immunodetection was performed using an enhanced chemiluminescence plus system (ECL plus, GE Healthcare UK) followed by autoradiography on Hyperfilm MP (Fujifilm Intelligent Dark Box LAS-3000 Luminoimage analyser, Japan).

In-gel enzyme digestion and protein identification by mass spectrometry. The two-dimensional gel was stained with Coomassie brilliant blue and pieces of gel corresponding to Western blot-positive spots were excised. Protein identification was done as previously reported (9). To remove the Coomassie brilliant blue staining, gel pieces were alternately washed with $25 \mathrm{mM}$ ammonium bicarbonate $/ 50 \%$ acetonitrile and finally dehydrated with acetonitrile. These pieces were completely dehydrated in a Speedvac device at $45^{\circ} \mathrm{C}$. Reduction was then performed by incubating the gel pieces at $56^{\circ} \mathrm{C}$ with $10 \mathrm{mM}$ DTT in $25 \mathrm{mM}$ ammonium bicarbonate for $45 \mathrm{~min}$. Alkylation was performed by placing the pieces in a shaker with $55 \mathrm{mM}$ iodoacetamide in $25 \mathrm{mM}$ ammonium bicarbonate for $30 \mathrm{~min}$ at room temperature in the dark. Gel pieces were again alternately washed with $25 \mathrm{mM}$ ammonium bicarbonate/50\% acetnitrile and dehydrated as before, then immersed in $20 \mu \mathrm{l}$ of L-(tosylamido-2-phenyl) ethyl chloromethyl ketonemodified trypsin $(20 \mathrm{ng} / \mu \mathrm{l}$; Promega, Madison, WI) on the shaker for $1 \mathrm{~h}$ at $4^{\circ} \mathrm{C}$. The excess trypsin was then discarded and the gel pieces were soaked in $25 \mathrm{mM}$ ammonium bicarbonate at $37^{\circ} \mathrm{C}$ overnight. Peptides were extracted by further shaking for 30 min followed by immersion in an ultrasonic bath for $3 \mathrm{~min}$ after which the supernatant was collected. The remaining gel was treated with $0.05 \%$ trifluoroacetic acid $/ 25 \%$ acetonitrile and the supernatant collected after shaking, then finally with $0.05 \%$ trifluoroacetic acid $/ 50 \%$ acetonitrile to complete the peptide extraction.

Extracts were then concentrated and desalted using a ZipTip $\mu$-C18 (Millipore, Billerica, MA). Extracts were loaded onto the MALDI target plate sandwiched by a fine layer of matrix solution prepared freshly everyday by dissolving $0.3 \mathrm{~g} / \mathrm{ml}$ of $\alpha$-cyano-4-hydroxycinnamic acid (Wako Purified Reagent, Kyoto, Japan) in acetone/ethanol (1:1, v/v). Measurements were taken using the Ultraflex MALDITOF/TOF mass spectrometer (Brucker Daltonics, Bremen, 
Germany) with an accelerating voltage of $20 \mathrm{kV}$. Laser wavelength was $337 \mathrm{~nm}$ and laser pulse frequency was $25 \mathrm{~Hz}$. The peptide mass fingerprint was used for protein identification from the tryptic fragment size using the Mascot Search engine based on the SwissProt protein database. The presence of peptides with a probable match was further confirmed by calculating the amino acid sequence by MSMS fragment analysis.

Detection of autoantibodies against $78 \mathrm{kDa}$ glucose-regulated protein (GRP78) in patient sera. Recombinant GRP78 was dot-blotted onto pieces of nitrocellulose membrane at $100 \mathrm{ng} /$ $2 \mu 1$ after which the membranes were blocked with $5 \%$ skimmed milk for $1 \mathrm{~h}$, washed with PBS Tween and incubated for $1 \mathrm{~h}$ with sera from patients with gastric cancer, esophageal cancer, colon cancer and healthy individuals diluted to 1:500. After further washings, the membranes were incubated with horseradish peroxidase-conjugated mouse anti-human immunoglobulin IgG (GE Healthcare UK) at 1:5000 dilution for $1 \mathrm{~h}$ and washed again. Immunodetection was performed again using an ECL plus followed by autoradiography on Hyperfilm MP.

Immunohistochemistry. Paraffin-embedded tissue sections were first deparaffinized with xylene and rehydrated with a graded ethanol series. Consecutively, the tissue sections were treated with $3 \%$ hydrogen peroxidase $(5 \mathrm{~min}$, room temperature) followed by incubation $\left(4^{\circ} \mathrm{C}\right.$, overnight $)$ with the 1:50 diluted goat anti-GRP78 antibody (N-20; Santa Cruz Biotechnology, CA) in a humidified chamber. After washing, anti-Sheep/goat Ig affinity isolated HRP conjugated raised in donkey (Chemicon, Australia) were applied for $1 \mathrm{~h}$ at room temperature. Finally, 3,3'-diaminobenzidine was used for color development and hematoxylin was used for counterstaining.

RNA isolation. Total RNA was isolated from frozen tumor tissue by an acid guanidium-phenol-chloroform method using Isogen (Nippon Gene, Toyama, Japan). Isolated total RNA pellets were dissolved in RNase-free, diethylpyrocarbonatetreated water. For first-strand cDNA synthesis, $5 \mu \mathrm{g}$ of total RNA, $1 \mu \mathrm{l}$ of oligo (dT)18 primer, and $6.5 \mu 1$ of Advantage RT-for-PCR kit (Clintech Laboratories, Palo Alto, CA), including $1 \mu \mathrm{l}$ of $\mathrm{dNTP}$ mix, $4 \mu \mathrm{l}$ of $5 \mathrm{X}$ reaction buffer and $0.5 \mu \mathrm{l}$ of RNase inhibitor and $1 \mu \mathrm{l}$ of reverse transcriptase (SuperScript II RNase H-Reverse Transcriptase, Invitrogen, Carlsbad, CA), were mixed to make a total volume of $20 \mu 1$. Reverse transcription reactions were incubated at $50^{\circ} \mathrm{C}$ for $90 \mathrm{~min}$. Finally, cDNA solution was diluted to a total volume of $100 \mu 1$.

Quantitative reverse transcription-PCR. Quantitative reverse transcription-PCR amplifications were done on a LightCycler quick system 330 (Roche Molecular Biochemicals, Mannheim, Germany). PCR conditions were initial denaturation at $95^{\circ} \mathrm{C}$ for $15 \mathrm{~min}$ followed by 40 cycles of denaturation at $95^{\circ} \mathrm{C}$ for $10 \mathrm{sec}$, annealing at $58^{\circ} \mathrm{C}$ for GRP78 or at $63^{\circ} \mathrm{C}$ for glucose6-phosphate dehydrogenase (G6PD) for $10 \mathrm{sec}$ and extension at $72^{\circ} \mathrm{C}$ for $10 \mathrm{sec}$. A standard curve was generated using fluorescent data from serial dilutions of the plasmid including a single PCR product. Data for GRP78 were normalized according to data for G6PD. All primers and probes used in this study were designed by Nihon Gene Research Laboratories (Sendai, Japan). Sequences of forward and reverse primers for GRP78 were 5'-GGGTTAGGTGTGTGTTCA-3' and 5'-CCCACACACGAGTATCTAGTCT-3', respectively. Sequences of hybridization probes $\mathrm{F}$ and $\mathrm{R}$ for GRP78 were 5'-GGGTCATGTGCATCTGGTGTAGGAAGTTTTTTCT-3' and 5'-CCATAAGTGACACCAATAAATGTTTGTTATTT ACACTGGTC-3', respectively. Sequences of forward and reverse primer for G6PD were 5'-TGGACCTGACCTACGG CAACAGATA-3' and 5'-GCCCTCATACTGGAAACCC-3', respectively. Sequences of hybridization probes $\mathrm{F}$ and $\mathrm{R}$ for G6PD were 5'-TTTTCACCCCACTGCTGCACC-3' and 5'-GATTGAGCTGGAGAAGCCCAAGC-3', respectively.

\section{Results}

Autoantibodies against gastric cancer cell line proteins in sera from patients with gastric cancer. Proteins from a mixture of three gastric cancer cell lines $(\mathrm{MkN}-1, \mathrm{MkN}-45$ and KATOIII) were separated by two-dimensional PAGE and visualized by Coomassie staining (Fig. 1A). To detect autoantibodies against proteins from gastric cancer cell lines, proteins were separated by two-dimensional PAGE and transferred onto PVDF membranes. Membranes were then incubated with sera from 10 patients with gastric cancer and 10 healthy control subjects. Each membrane was treated with one serum sample for the primary antibody reaction and then with horseradish peroxidase-conjugated mouse anti-human immunoglobulin as the secondary antibody reaction. Sera from patients with gastric cancer reacted as multiple spots (Fig. 1B), whereas some reactive protein spots were observed in healthy controls and were considered as non-specific reactivity. One of reactive spots, with an approximate isoelectric point 5.0 and molecular weight of $80 \mathrm{kDa}$, was observed only in patients with gastric cancer (Fig. 1C) and not in healthy controls (Fig. 1D).

Identification of the reactive protein as $78 \mathrm{kDa}$ glucoseregulated protein (GRP78). The reactive spot shown in Fig. 1 was excised from a Coomassie stained gel and peptides were extracted after in-gel enzyme digestion and analyzed using Ultraflex MALDI-TOF/TOF mass spectrometry. The acquired spectra were processed and searched against a Mascot Search engine based on the SwissProt protein databases (Fig. 2). The protein was identified as $78 \mathrm{kDa}$ glucose-regulated protein (GRP78), which comprises of 218 amino acids with a predicted molecular weight of $72.4 \mathrm{kDa}$ and a theoretical isoelectric point of 4.9 (Fig. 1A). Identification as GRP78 was confirmed by Western blotting using goat anti-GRP78 antibody (N-20; Santa Cruz Biotechnology) (Fig. 1E).

Confirmation of antibodies against GRP78 in sera from patients with gastric cancer, patients with other cancers and healthy individuals by Western blotting. To screen for antoantibodies against GRP78 in sera from patients with gastric cancer, other cancers (esophageal cancer and colon cancer) and from healthy individuals, Western blotting of recombinant GRP78 protein (100 ng) and immunoreactions with sera from 60 patients with gastric cancer, 30 patients with 

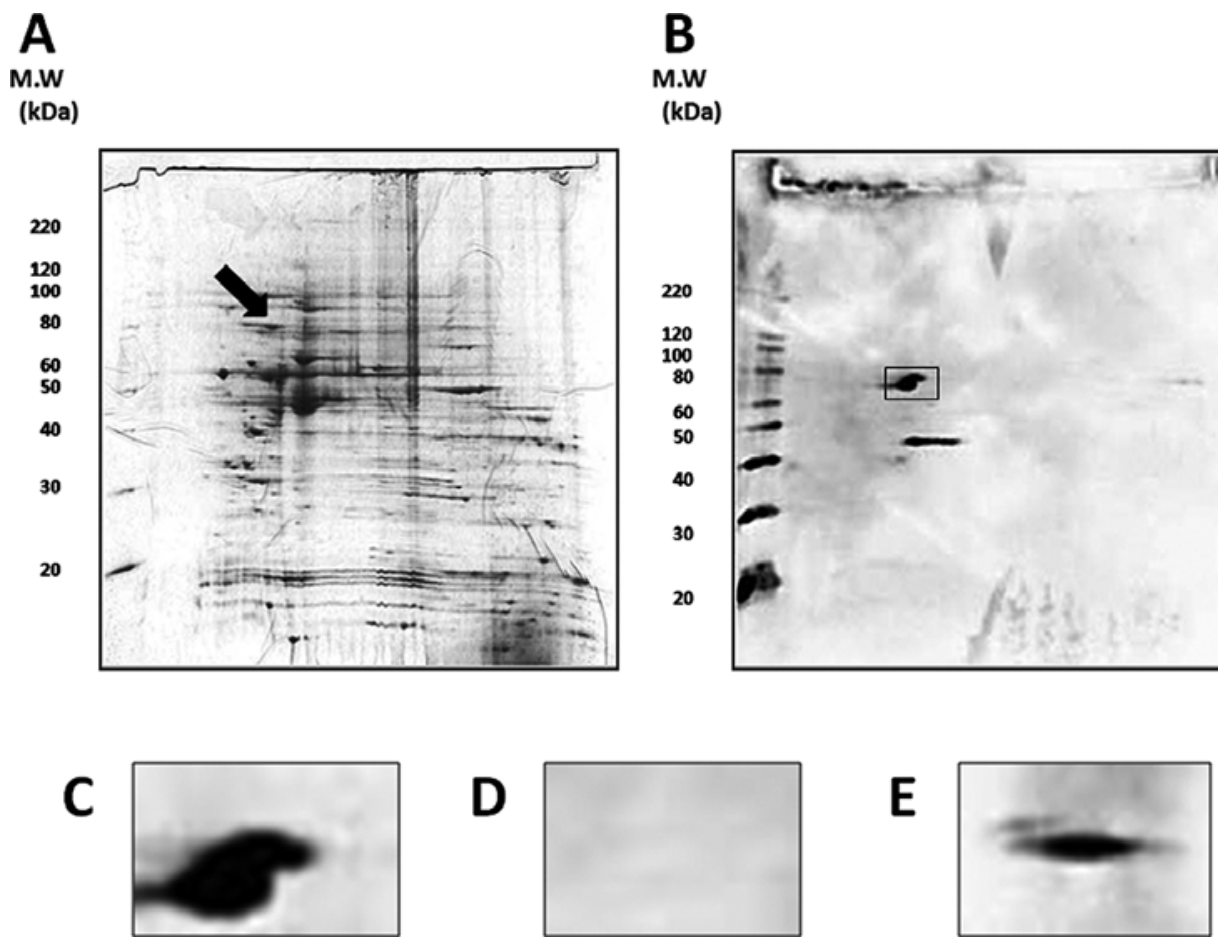

Figure 1. GRP78 autoantibodies in the serum from a patient with gastric cancer. (A) Total protein extracts from a mixture of gastric cancer cell lines MkN-1, MkN-45 and KATOIII were separated by two-dimensional PAGE and visualized by Coomasie brilliant blue staining. (B) Protein lysates from gastric cancer cell lines MkN-1, MkN-45 and KATOIII were separated by two-dimensional PAGE, transferred onto PVDF membranes and incubated with diluted sera (1:200) from patients with gastric cancer. (C) Enlargement of the region containing the GRP78 (box in B). (D) PVDF membrane incubated with sera from healthy individuals. (E) PVDF membranes incubated with anti-GRP78 antibodies (1:2000). PVDF membranes were incubated with appropriate secondary antibodies and visualized by chemiluminescence.

other cancers (esophageal cancer, $\mathrm{n}=15$; colon cancer, $\mathrm{n}=15$ ) and 20 healthy individuals was performed (Table I). Reactivity was demonstrated in 17 of 60 (28.3\%) patients with gastric cancer (Fig. 3A) and 0 of $20(0.0 \%)$ healthy individuals (Fig. 3B). Autoantibodies against GRP78 were found in sera of 4 of $15(26.7 \%)$ patients with esophageal cancer and 3 of $15(20.0 \%)$ patients with colon cancer. In gastric cancer patients, reactivity was recognized for all stages of disease $(25.9 \%$ in stage I, 33.3\% in stage II, 28.6\% in stage III and $33.3 \%$ in stage IV). There was no correlation between appearance of autoantibodies against GRP78 and disease stage.

Expression of GRP78 $m R N A$. To examine whether the immunogenicity of GRP78 in gastric cancer could be due to elevated transcription mechanism, tissue expression of GRP78 mRNA was compared between patients with and without autoantibodies against GRP78. To examine GRP78 mRNA expression in 18 tissue samples (with autoantibodies, $\mathrm{n}=7$; without autoantibodies, $\mathrm{n}=11$ ), real-time reverse transcriptionPCR was done with expression levels of G6PD as an internal control. After normalization, the GRP78/G6PD ratio was calculated in each group.

The level of GRP78 mRNA expression tended to be higher in patients with autoantibodies than in patients without autoantibodies although the difference did not reach statistical significance $(\mathrm{P}=0.06)$ (data not shown). These findings suggest that overexpression of GRP78 contributes to immunogenicity.
Table I. GRP78 autoantibody in patients sera.

\begin{tabular}{lcc}
\hline Subjects & No. subjects & $\begin{array}{c}\text { GRP78 autoantibody- } \\
\text { positive }\end{array}$ \\
\hline Gastric cancer & 60 & $17(28.3 \%)$ \\
Stage I & 27 & $7(25.9 \%)$ \\
Stage II & 3 & $1(33.3 \%)$ \\
Stage III & 21 & $6(28.6 \%)$ \\
Stage IV & 9 & $3(33.3 \%)$ \\
Other type of cancer & 30 & $7(23.3 \%)$ \\
Esophageal cancer & 15 & $4(26.7 \%)$ \\
Colon cancer & 15 & $3(20.0 \%)$ \\
Other control & & \\
Healthy subjects & 20 & $0(0.0 \%)$ \\
\hline
\end{tabular}

Immunohistochemistry for GRP78. GRP78 expression levels in gastric cancer tissue and normal gastric mucosa were assessed by immunohistochemistry with goat anti-GRP78 antibody (N-20; Santa Cruz Biotechnology). In normal gastric mucosa, GRP78 positivity was occasionally observed in the deep propria glands, but not in the superficial epithelium (Fig. 4A and B). In gastric cancer tissue, GRP78 was expressed at high levels in the cytoplasm of cancer cells regardless of the depth from the surface or histological differentiation (Fig. 4C and D). 

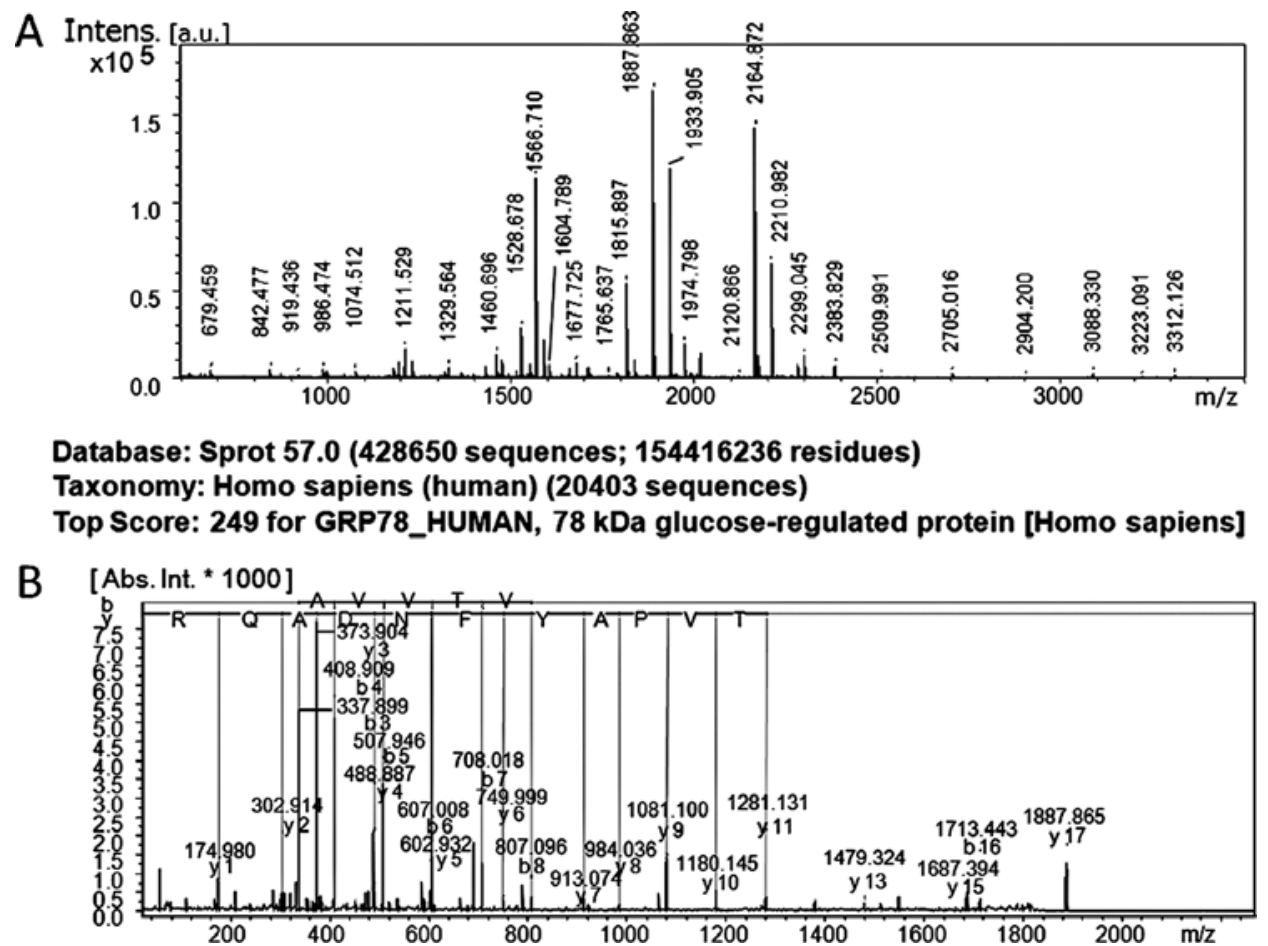

Figure 2. (A) Peptides were extracted from excised gel pieces after in-gel enzyme digestion and analyzed using Ultraflex MALDI-TOF/TOF mass spectrometer. Acquired spectra were processed and searched against a Mascot Search engine based on the SwissProt protein databases. The reactive spot was identified as GRP78. (B) Tandem mass (MALDI-TOF/TOF) spectrum of one peptide obtained from a tryptic digest, $\mathrm{MH}^{+}=1187.863(165-181)$, indicating confident identification of GRP78.

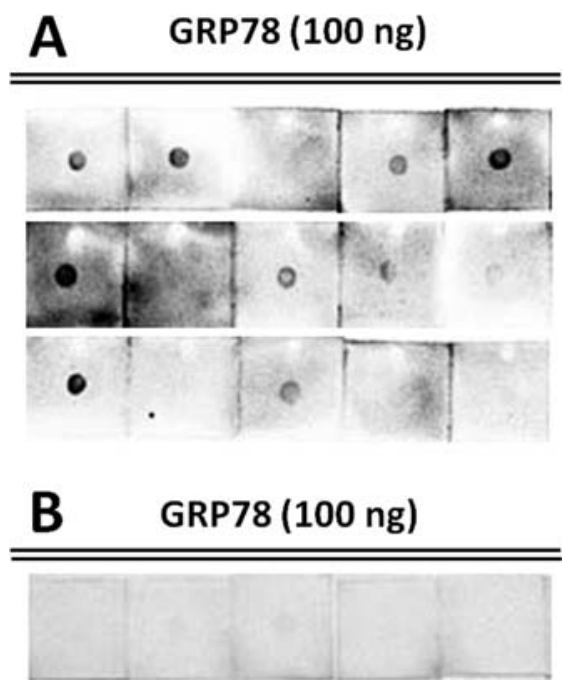

Figure 3. (A) Recombinant GRP78 (100 ng) was analyzed by Western blotting and reacted with sera from 60 patients with gastric cancer. Reactivity to GRP78 was shown in 17 of $60(28.3 \%)$ patients with gastric cancer. (B) Recombinant GRP78 (100 ng) was analyzed by Western blotting and reacted with sera from 20 healthy subjects. Reactivity directed against GRP78 was found in 0 of $20(0.0 \%)$ healthy individuals.

\section{Discussion}

We have implemented a proteomics-based approach to identify proteins that elicit a humoral response in gastric cancer patients. This approach allows screening by Western blotting of patient sera for antibodies that react against separated tumor cell proteins. In this study, we have focused on a search for autoantibodies against gastric cancer protein in the MkN-1, MkN-45 and KATOIII cell lines. Through this, we have identified $78 \mathrm{kDa}$ glucose-regulated protein (GRP78) as a candidate antigen and recognized autoantibodies against GRP78 by Western blotting. A humoral response directed against GRP78 occurred in $28.3 \%$ of gastric cancer patients, $26.7 \%$ of esophageal cancer patients, $20.0 \%$ of colon cancer patients and in $0.0 \%$ of healthy individuals. In gastric cancer, the positivity for autoantibodies against GRP78 was $25.9 \%$ in stage I, $33.3 \%$ in stage II, $28.6 \%$ in stage III and $33.3 \%$ in stage IV. Autoantibodies against GRP78 occurred at similar rates in malignancies of the gastrointestinal tract, and in gastric cancer, positivity was observed regardless of disease stage. Hence, autoimmunity was found to have no correlation with tumor progression.

Discovered in the late 1970s (10) and also known as immunoglobulin heavy chain binding protein, GRP78 resides primarily within the endoplasmic reticulum of virtually all cells (11-13). It shares 60\% amino acid sequence homology with Heat Shock Protein 70 (HSP70) and is therefore considered a member of the HSP70 family of molecular chaperones $(12,13)$. As a chaperone, GRP78 transiently associates with nascent polypeptides to facilitate folding and assembly and prevent deleterious aggregations (14). Although GRP78 is constitutively expressed, its expression is enhanced up to 20-fold in cells under a variety of stressful conditions which interfere with protein glycosylation, folding, transport and calcium homeostasis $(14,15)$. Because of poor vascularization, solid tumors usually contain regions undergoing glucose deprivation and hypoxia resulting in acidosis 
A

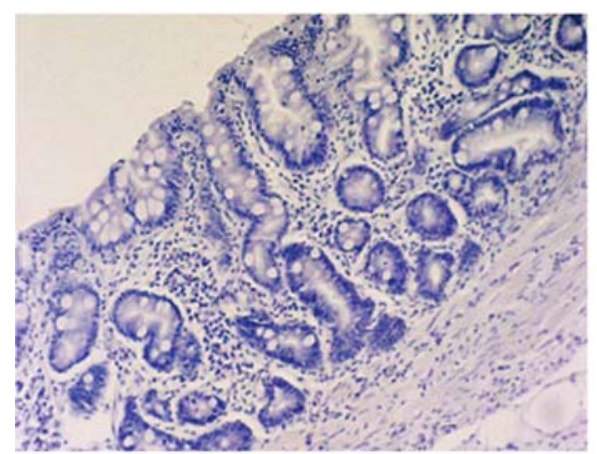

C

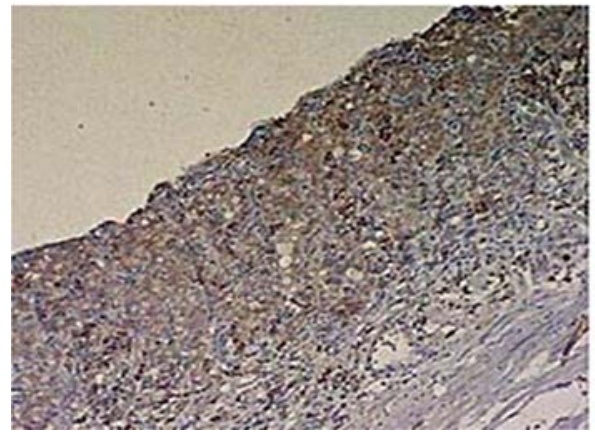

B

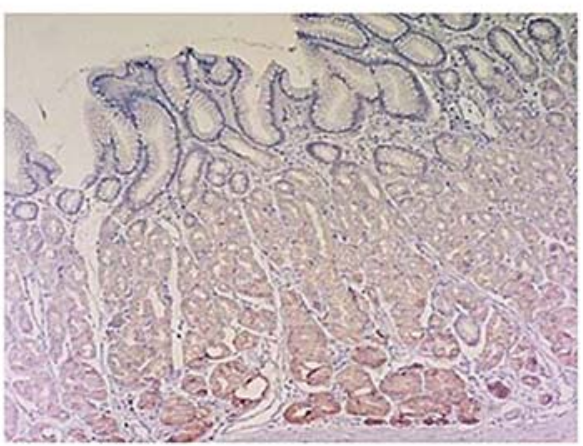

D

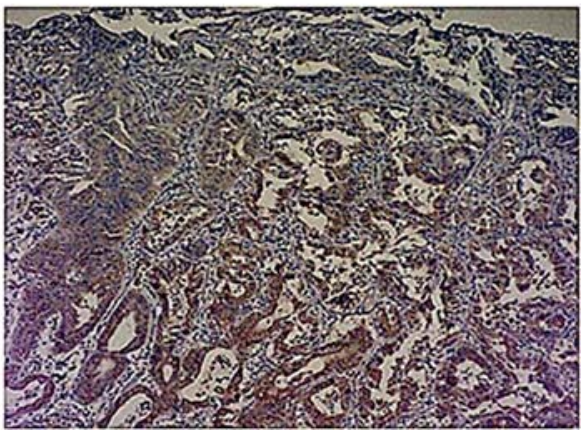

Figure 4. GRP78 expression in cancer and normal gastric mucosa as assessed by immunohistochemistry with goat anti-GRP78 antibodies. (A and B) In normal gastric epithelium, GRP78 positivity was occasionally observed in the deep propria glands, but not in the superficial epithelium. (C and D) In early and advanced gastric cancer tissue, GRP78 was highly expressed in the cytoplasm of cancer cells, tumor grades were poorly differenciated adenocarcinoma and well differenciated adenocarcinoma, respectively. The expression of GRP78 was found regardless of the depth from the surface and histological differentiation $(\mathrm{x} 100)$.

and alterations in cell metabolism (16). The endoplasmic reticulum responds to these stresses by evoking specific signal transduction pathways that protect against stress-induced apoptosis. One pathway, designated as the unfolded protein response pathway, induces GRP78 as a result of accumulation of malfolded proteins in the endoplasmic reticulum $(14,17)$. Investigations of GRP78 induction and function have revealed the mechanisms by which GRP78 facilitates survival of cancer cells.

Expression of GRP78 in malignant tissues has been reported frequently. High levels of expression have been found in a variety of malignancies including breast, lung, prostate and hepatocellular carcinoma, and more recently in gastric cancer (18-22). It has also been suggested that GRP78 expression correlates with higher pathological grade, recurrence and poor prognosis, mainly through immunohistochemical and clinical studies $(20,23,24)$. In 2003 , both overexpression of and autoantibodies against GRP78 in prostate cancer was first reported and correlated with poor prognosis and aggressive tumor behavior $(25,26)$. In the present study, we discovered autoantibodies against GRP78 in gastric cancer using a protemics-based approach. Similar to other accounts in the literature $(20,23,24)$, we observed overexpression of GRP78 in the cytoplasm of gastric cancer cells compared to non-cancerous gastric epithelium by immunohistochemistry. We also assessed the correlation between the presence of autoantibodies and several histological factors including tumor grade, tumor depth, lymphatic and vascular invasion. Antibody positivity was present equally in all stages of gastric cancer and there were no significant differences in histological factors between antibody-positive and antibody-negative patients (data not shown). Therefore, no conclusions can be drawn about the mechanisms underlying the generation of immunity against GRP78 as antibodies seem to be generated regardless of tumor progression. These results suggest that the detection of autoantibodies against GRP78 may also be possible during the early stage of gastric cancer.

As previously reported, the presence of antibodies may be related to tumor behavior. On the other hand, to examine whether the immunogenicity of GRP78 in gastric cancer could be due to elevated transcription mechanism, expression levels of GRP78 mRNA in the tumor tissue was compared between patients with and without autoantibodies against GRP78. The level of GRP78 mRNA expression tended to be higher in patients with autoantibodies than in patients without autoantibodies $(\mathrm{P}=0.06)$ although the difference did not reach statistical significance. The mechanisms underlying immunogenicity is not entirely understood (27). It has been suggested that the immunogenicity of tumor proteins may depend on the level of expression, post-translational modification, or other types of processing of the protein, the extent of which may vary among tumors of a similar type. Soussi (28) has shown that p53 antibodies are found predominantly in human cancer patients, and such antibodies are associated with p53 gene missense mutations and p53 accumulation in the tumor. As no significant difference in GRP78 expression was observed between autoantibody-positive and -negative patients in the present study, immunogenicity may in turn be due to a 
mutation of the GRP78 gene instead of overexpression. Further examination is needed to consider the mechanism of the generation of GRP78 autoantibodies in gastric cancer, such as quantitative analysis of autoantibodies against GRP78 by ELISA or the identification of GRP78 gene missense mutations in gastric cancer patients.

Since its discovery, many researchers have reported that GRP78 facilitates tumor progression in malignant cells. GRP78 has anti-apoptotic functions in the stressed malignant cells and elucidating this will lead to the establishment of targeted anti-cancer therapies. Misra et al (29) reported the effects on p53 of down-regulating the function of cell surface-associated GRP78 by antibodies directed against the $\mathrm{COOH}$-terminal domain of GRP78. The fundemental role of GRP78 in tumor promotion was first shown by inhibiting GRP78 induction in fibrosarcoma cells with antisense, which rendered them completely incapable of forming tumors in vivo (30-32). Delivery of a suicide transgene driven by the GRP78 promoter into fibrosarcoma or breast tumor cells also completely blocks formation of sizable tumors in mice $(30,31)$. The role of GRP78 in the progression of cancer is also apparent through the effects of targeting plasma membrane GRP78 levels in heterozygous mice impeding tumor progression through multiple mechanisms (33). Reddy et al (34) have reported that induction of GRP78 correlates with the resistance to topoisomerase-directed drugs such as etoposide and camptothecin by reducing apoptosis. These studies indicate that GRP78-associated signaling pathways are important in the regulation of apoptotic cell death and that GRP78 could be an attractive therapeutic target for solid tumors. In the present study, GRP78 immuno-reactivity was observed in sera from not only the gastric cancer patients but also esophageal and colon cancer patients at a similar rate. As with prostate cancer, studies investigating the role of GRP78 in malignancies of gastrointestinal tract are needed. Likewise, further knowledge about the role of GRP78 in cancer in general may lead to the development of anti-cancer treatments applicable to different types of cancers.

In conclusion, we have for the first time identified autoantibodies against GRP78 in sera from patients with gastric cancer. The detection of this humoral response may be useful not only as a tumor marker, but also to guide effective new immunotherapies. Further investigation is needed to evaluate the sensitivity and specificity of autoantibodies against GRP78 in large-scale validation studies and to consider the mechanism underlying the generation of immunity against GRP78 for gastric cancer. In addition, the roles of autoantibodies against GRP78 and the GRP78 protein itself in relation to the proliferation of tumor cells in gastric cancer needs to be explored further. As regards to the detection of novel antoantibodies, the proteomics-based approach that we implemented here offers a powerful tool to identify novel proteins that may display immunogenicity in cancer patients which may be of important clinical use in the future.

\section{References}

1. Brichory FM, Misek DE, Yim AM, et al: An immune response manifested by the common occurrence of Annexin I and II autoantibodies and high circulating levels of IL-6 in lung cancer. Proc Natl Acad Sci USA 98: 9824-9829, 2001.
2. Brichory F, Beer D, Le Naour F, Giordano T and Hanash S: Proteomics-based identification of protein gene product 95 as a tumor antigen that induces a humoral immune response in lung cancer. Cancer Res 61: 7908-7912, 2001.

3. Takashima M, Kuramitsu Y, Yokoyama Y, et al: Proteomics analysis of autoantibodies in patients with hepatocellular carcinoma. Proteomics 6: 3894-3900, 2006.

4. Li L, Chen SH, Yu CH, Li YM and Wang SQ: Identification of hepatocellular-carcinoma-associated antigens and autoantibodies by serological proteome analysis combined with protein microarray. J Proteome Res 7: 611-620, 2008.

5. Fujita Y, Nakanishi T, Hiramatsu M, et al: Proteomics-based approach identifying autoantibody against peroxiredoxin VI as a novel serum marker in esophageal squamous cell carcinoma. Clin Cancer Res 12: 6415-6420, 2006.

6. Fujita Y, Nakanishi T, Miyamoto Y, et al: Proteomics-based identification of autoantibody against heat shock protein 70 as a diagnostic marker in esophageal squamous cell carcinoma. Cancer Lett 263: 280-290, 2008.

7. Pavoni E, Pucci A, Vaccaro P, et al: A study of the humoral immune response of breast cancer patients to a panel of human tumor antigens identified by phage display. Cancer Detect Prev 30: 248-256, 2006.

8. Le Naour F, Misek DE, Krause MC, et al: Proteomics-based identification of RS/DJ-1 as a novel circulating tumor antigen in breast cancer. Clin Cancer Res 7: 3328-3335, 2001.

9. Nakanishi T, Koyama R, Ikeda T and Shimizu A: Catalogue of soluble proteins in the human vitreous humor: comparison between diabetic retinopathy and macular hole. J Chromatogr B Analyt Technol Biomed Life Sci 776: 89-100, 2002.

10. Baldwin GS, Chandler R, Seet KL, et al: Structural studies on a $75-\mathrm{kDa}$ glycoprotein isolated from porcine gastric mucosal membranes: close homology with the 78-kDa glucose-regulated family of proteins. Protein Seq Data Anal 1: 7-12, 1987.

11. Little E, Ramakrishnan M, Roy B, Gazit G and Lee AS: The glucose-regulated proteins (GRP78 and GRP94): functions, gene regulation, and applications. Crit Rev Eukaryot Gene Expr 4: 1-18, 1994.

12. Haas IG: BiP (GRP78), an essential hsp70 resident protein in the endoplasmic reticulum. Experientia 50: 1012-1020, 1994

13. Munro S and Pelham HR: An Hsp70-like protein in the ER: identity with the $78 \mathrm{kd}$ glucose-regulated protein and immunoglobulin heavy chain binding protein. Cell 46: 291-300, 1986.

14. Lee AS: Mammalian stress response: induction of the glucoseregulated protein family. Curr Opin Cell Biol 4: 267-273, 1992.

15. Li WW, Alexandre S, Cao X and Lee AS: Transactivation of the GRP78 promoter by $\mathrm{Ca}^{2+}$ depletion. A comparative analysis with A23187 and the endoplasmic reticulum $\mathrm{Ca}(2+)$ ATPase inhibitor thapsigargin. J Biol Chem 268: 12003$12009,1993$.

16. Brown JM and Giaccia AJ: The unique physiology of solid tumors: opportunities (and problems) for cancer therapy. Cancer Res 58: 1408-1416, 1998.

17. Yoshida H, Matsui T, Yamamoto A, Okada T and Mori K: XBP1 mRNA is induced by ATF6 and spliced by IRE1 in response to ER stress to produce a highly active transcription factor. Cell 107: 881-891, 2001.

18. Fernandez PM, Tabbara SO, Jacobs LK, et al: Overexpression of the glucose-regulated stress gene GRP78 in malignant but not benign human breast lesions. Breast Cancer Res Treat 59: 15-26, 2000.

19. Shuda M, Kondoh N, Imazeki N, et al: Activation of the ATF6, XBP1 and grp78 genes in human hepatocellular carcinoma: a possible involvement of the ER stress pathway in hepatocarcinogenesis. J Hepatol 38: 605-614, 2003.

20. Zhang J, Jiang Y, Jia Z, et al: Association of elevated GRP78 expression with increased lymph node metastasis and poor prognosis in patients with gastric cancer. Clin Exp Metastasis 23: 401-410, 2006.

21. Daneshmand S, Quek ML, Lin E, et al: Glucose-regulated protein GRP78 is up-regulated in prostate cancer and correlates with recurrence and survival. Hum Pathol 38: 1547-1552, 2007.

22. Uramoto H, Sugio K, Oyama T, et al: Expression of endoplasmic reticulum molecular chaperone Grp78 in human lung cancer and its clinical significance. Lung Cancer 49: 55-62, 2005.

23. Fu Y and Lee AS: Glucose regulated proteins in cancer progression, drug resistance and immunotherapy. Cancer Biol Ther 5: 741-744, 2006 
24. Zheng HC, Takahashi H, Li XH, et al: Overexpression of GRP78 and GRP94 are markers for aggressive behavior and poor prognosis in gastric carcinomas. Hum Pathol 39: 1042-1049, 2008.

25. Mintz PJ, Kim J, Do KA, et al: Fingerprinting the circulating repertoire of antibodies from cancer patients. Nat Biotechnol 21: $57-63,2003$

26. Gonzalez-Gronow M, Cuchacovich M, Llanos C, Urzua C, Gawdi G and Pizzo SV: Prostate cancer cell proliferation in vitro is modulated by antibodies against glucose-regulated protein 78 isolated from patient serum. Cancer Res 66: 11424-11431, 2006.

27. Zinkernagel RM: What is missing in immunology to understand immunity? Nat Immunol 1: 181-185, 2000.

28. Soussi T: p53 Antibodies in the sera of patients with various types of cancer: a review. Cancer Res 60: 1777-1788, 2000.

29. Misra UK, Mowery Y, Kaczowka S and Pizzo SV: Ligation of cancer cell surface GRP78 with antibodies directed against its $\mathrm{COOH}$-terminal domain up-regulates p53 activity and promotes apoptosis. Mol Cancer Ther 8: 1350-1362, 2009.
30. Li J and Lee AS: Stress induction of GRP78/BiP and its role in cancer. Curr Mol Med 6: 45-54, 2006.

31. Lee AS: GRP78 induction in cancer: therapeutic and prognostic implications. Cancer Res 67: 3496-3499, 2007.

32. Fu Y, Li J and Lee AS: GRP78/BiP inhibits endoplasmic reticulum BIK and protects human breast cancer cells against estrogen starvation-induced apoptosis. Cancer Res 67: 3734-3740, 2007.

33. Dong D, Ni M, Li J, et al: Critical role of the stress chaperone GRP78/BiP in tumor proliferation survival and tumor angiogenesis in transgene-induced mammary-tumor development. Cancer Res 68: 498-505, 2008.

34. Reddy RK, Mao C, Baumeister P, Austin RC, Kaufman RJ and Lee AS: Endoplasmic reticulum chaperone protein GRP78 protects cells from apoptosis induced by topoisomerase inhibitors: role of ATP binding site in suppression of caspase-7 activation. J Biol Chem 278: 20915-20924, 2003. 